\title{
Effects of dimethyl fumarate on neuroprotection and immunomodulation
}

\author{
Philipp Albrecht ${ }^{1}$, Imane Bouchachia ${ }^{1}$, Norbert Goebels ${ }^{1}$, Nadine Henke ${ }^{1}$, Harald H Hofstetter ${ }^{1}$, Andrea Issberner ${ }^{1}$, \\ Zsuzsa Kovacs', Jan Lewerenz², Dmitrij Lisak', Pamela Maher ${ }^{3}$, Anne-Kathrin Mausberg ${ }^{1}$, Kim Quasthoff', \\ Corinna Zimmermann ${ }^{1}$, Hans-Peter Hartung ${ }^{1}$ and Axel Methner ${ }^{1,4^{*}}$
}

\begin{abstract}
Background: Neuronal degeneration in multiple sclerosis has been linked to oxidative stress. Dimethyl fumarate is a promising novel oral therapeutic option shown to reduce disease activity and progression in patients with relapsing-remitting multiple sclerosis. These effects are presumed to originate from a combination of immunomodulatory and neuroprotective mechanisms. We aimed to clarify whether neuroprotective concentrations of dimethyl fumarate have immunomodulatory effects.

Findings: We determined time- and concentration-dependent effects of dimethyl fumarate and its metabolite monomethyl fumarate on viability in a model of endogenous neuronal oxidative stress and clarified the mechanism of action by quantitating cellular glutathione content and recycling, nuclear translocation of transcription factors, and the expression of antioxidant genes. We compared this with changes in the cytokine profiles released by stimulated splenocytes measured by ELISPOT technology and analyzed the interactions between neuronal and immune cells and neuronal function and viability in cell death assays and multi-electrode arrays. Our observations show that dimethyl fumarate causes short-lived oxidative stress, which leads to increased levels and nuclear localization of the transcription factor nuclear factor erythroid 2-related factor 2 and a subsequent increase in glutathione synthesis and recycling in neuronal cells. Concentrations that were cytoprotective in neuronal cells had no negative effects on viability of splenocytes but suppressed the production of proinflammatory cytokines in cultures from C57BL/6 and SJL mice and had no effects on neuronal activity in multi-electrode arrays.
\end{abstract}

Conclusions: These results suggest that immunomodulatory concentrations of dimethyl fumarate can reduce oxidative stress without altering neuronal network activity.

Keywords: Dimethyl fumarate, Oxidative stress, Neuroprotection, Neuromodulation

\section{Background}

Chronic disability in multiple sclerosis (MS) is due to neuronal degeneration, which is not amenable, or is incompletely amenable to immunomodulatory therapy. The mechanisms remain elusive, but there is accumulating evidence that oxidative stress may play a key role [1-3]. Dimethyl fumarate (DMF) is a novel oral therapeutic agent which reduces disease activity and progression in patients with relapsing-remitting MS [4,5]. Previously

\footnotetext{
* Correspondence: axel.methner@gmail.com

'Department of Neurology, Heinrich-Heine-University Düsseldorf, Medical Faculty, Düsseldorf 40225, Germany

${ }^{4}$ Neurologische Klinik der Heinrich-Heine-Universität Düsseldorf, Moorenstr. 5, D-40225, Düsseldorf 40225, Germany

Full list of author information is available at the end of the article
}

suggested immunomodulatory mechanisms of action of DMF or its metabolite monomethyl fumarate (MMF) include inhibition of cytokine-induced nuclear translocation of the nuclear factor kappa B (NF-kB) [6], apoptosis of stimulated T cells [7], and increased production of the TH2 cytokines IL-4 and IL- 5 in stimulated T cells, whereas generation of the TH1 cytokine interferon gamma (IFN- $\gamma$ ) [8] is supposed to remain unaffected. DMF also activates the transcription factor Nrf2 (nuclear factor erythroid 2-related factor 2), which binds to antioxidant response elements in the promoters of protective genes such as NADPH-quinone-oxidoreductase-1 (NQO1) [9] and heme-oxygenase-1 [10]. This ultimately raises the levels of the important intracellular antioxidant

\section{Biomed Central}


glutathione $[9,10]$. However, short-term incubation with DMF for 60 minutes decreases the glutathione content of cortical primary cultures and OLN-93 cells [11,12].

Here, we first investigated the concentration and time dependence of DMF-mediated protection in neuronal cells using a model of endogenous oxidative stress, oxidative glutamate toxicity, where extracellular glutamate blocks the glutamate-cystine antiporter system Xc-. This leads to deprivation of cystine and its reduced form cysteine, the rate-limiting substrate for the synthesis of glutathione. The subsequent glutathione depletion gives rise to the accumulation of reactive oxygen species and cell death by oxidative stress (recently reviewed [13]). We show herein that neuroprotective concentrations of DMF suppress cytokine production by splenocytes from two different mouse strains without effecting apoptosis and do not impact neuronal network activity studied with dissociated cortical cultures grown on multielectrode arrays [14] which allows a highly sensitive and reproducible assessment of network activity. Our results suggest that low doses of DMF may promote cellular resistance against oxidative stress and cause immunomodulation independent of $\mathrm{T}$ cell apoptosis or alterations in endogenous brain activity.

\section{Materials and methods Material}

DMF and MMF (sodium salt) for all experiments were obtained from Biogen Idec, Carl-Zeiss-Ring 685737 Ismaning, Germany and solubilized in dimethylsulfoxide (DMSO), which was also used as the vehicle control. The $\mathrm{pH}$ of all media was kept constant at 7.4. Cell culture dishes were from Greiner Bio-One, Maybachstraße 2., 72636 Frickenhausen, Germany; DMEM cell culture medium, sterile phosphate buffered saline were from PAA, Unterm Bornrain 2, 35091 Cölbe, Germany; penicillin, streptomycin were from Gibco/Life Technologies, Frankfurter Straße 129B, 64293 Darmstadt, Germany; cryopreserved primary dissociated cortical cultures from embryonic rats were from QBM Cell Science Inc., 1200 Montreal Road, Building M23A, Suite 147, Ottawa, Ontario, Canada; Cell Titer Blue was from Promega, Schildkrötstraße 1568199 Mannheim, Germany; the high contact imaging microscope, the anti-CD3 antibody, 7-AAD and Annexin V PE were from Becton Dickinson, Tullastr. 8-12, 69126 Heidelberg, Germany; the anti-Nrf2 antibody was from Santa Cruz Biotechnology, Bergheimer Straße 89, 69115 Heidelberg, Germany; the antiActin antibody and secondary antibodies were from Millipore, 290 Concord Road Billerica, MA 01821, USA; the anti-NF- $k B$-antibody was from Cell Signaling Technologies, 3 Trask Lane Danvers, MA 01923, USA; multielectrode arrays were from Multichannel Systems, Aspenhaustrasse 21. 72770 Reutlingen, Germany; the
MEA analyzing software Spanner was from RESULT software, 47918 Tönisvorst, Germany; the Universal Probe Library $^{\text {TM }}$ was from Roche, Emil-Barell-Str. 179639 Grenzach-Wyhlen, Germany; Fam-Tamra labeled oligonucleotides were from Eurofins-MWG-Operon, Anzingerstr. 7a, 85560 Ebersberg, Germany; the TNF $\alpha$ ELISA was from R\&D Systems, Borsigstrasse 7. 65205 Wiesbaden, Germany; the Immunospot Analyzer was from CTL, 2860 Fisher Road, Columbus, OH 43204, USA; Prism software was from GraphPad Software, 2236 Avenida de la Playa, La Jolla, CA 92037, USA; spreadsheet software was from Microsoft, Konrad-Zuse-Str. 1, 85716 Unterschleißheim, Germany; all other chemicals were from Sigma Aldrich, Georg-Heyken-Str. 14 D-21147 Hamburg Germany.

\section{Cell culture, viability assays and glutathione measurement}

The preparation of embryonic primary cortical cultures and splenocyte cultures from C57BL/6 and SJL mice and the cell culture of HT22 and fibroblast cells were performed as described $[15,16]$. For the analysis of network activity, cryopreserved primary dissociated cortical cultures from embryonic rats (embryonic day 18, E18, QBM Cell Science) were employed. After thawing, the cells were plated at a final density of $10^{5}$ cells on PDL/laminin-coated multi-electrode arrays (MEAs) or coverslips. Neuronal cultures were incubated in a humidified atmosphere $\left(5 \% \mathrm{CO}_{2} / 95 \%\right.$ air $)$ at $37{ }^{\circ} \mathrm{C}$ for $24 \mathrm{~h}$ in DMF or vehicle prior to glutamate treatment. Viability was quantitated $24 \mathrm{~h}$ after glutamate addition by the Cell Titer Blue (CTB) assay (Promega) and normalized to vehicle treatment. Total glutathione was measured enzymatically as described previously [15] and normalized to cellular protein measured by the bicinchoninic acidbased method (Pierce). Glutathione released into the cell culture medium was also quantitated enzymatically after $4 \mathrm{~h}$ in cystine-free medium and normalized to total cellular protein. Cell viability of splenocytes was assessed using flow cytometry quantitating 7-AAD (BD Pharmingen \#51-68981E) and Annexin V PE (BD Pharmingen \#556421) stained cells according to manufacturers' protocols.

\section{Cell fractionation, SDS-PAGE and immunoblotting}

Differential detergent fractionation and immunoblotting were performed as previously described [15] using antiNrf2 (1:1000; Santa Cruz Biotechnology; \#SC13032) and anti-Actin (1:3000; Millipore, MAB1501) antibodies.

\section{Translocation analysis of NF-kB and Nrf2}

Intracellular localization of transcription factors was quantitated by high-content imaging using a BD Pathway 855 microscope (BD Biosciences). HT22 cells were fixed 
with $4 \%$ paraformaldehyde and permeabilized with $0.3 \%$ Triton-X 100, blocked with Roti ImmunoBlock (Roth \#144.1) for $1 \mathrm{~h}$ before they were incubated with primary antibodies (NF-kB: Cell Signaling \#9936 S, Nrf2: Santa Cruz \#sc-13032) overnight and stained with secondary fluorochrome-labeled antibodies (Millipore \#AP132F and $\# 124 \mathrm{~F}$ ). Fluorescence intensities in regions of interest defined by the nuclear stain DAPI $(150 \mathrm{nM})$ were compared with those of a second concentric band surrounding the nuclei and corresponding to the cytoplasm.

\section{Quantitative real-time PCR}

RNA extraction, reverse transcription and quantitative real-time PCR were performed as previously described [15] using Fam/Dark-quencher probes from the Universal Probe Library ${ }^{\mathrm{TM}}$ (Roche) or individually designed Fam/Tamra probes (MWG). Beta-actin and HPRT served as endogenous control genes and showed no differential expression after incubation with DMF. Primer and probe sequences can be obtained from the authors.

\section{TNFa ELISA}

Primary splenocytes from 6- to 8-week old female C57BL/6 and SJL mice were stimulated with $1 \mu \mathrm{g} / \mathrm{ml}$ anti-CD3 (BD Bioscience) and treated with 1, 10 and $100 \mu \mathrm{M}$ of DMF. Supernatants were collected after $48 \mathrm{~h}$ and concentration of TNF $\alpha$ was measured following the manufacturer's protocol (R\&D Systems).

\section{Cytokine enzyme-linked immunosorbent spot (ELISPOT) assays}

ELISPOT assays were essentially performed as previously described [16] using splenocytes from 6- to 8-week old female C57BL/6 and SJL mice. Splenocytes were incubated with $10 \mu \mathrm{M}$ DMF or vehicle for $24 \mathrm{~h}$ while stimulated with $0.5 \mu \mathrm{g} / \mathrm{ml}$ anti-mouse CD3. Computerized ELISPOT analysis was done using an Immunospot Analyzer (CTL).

\section{Extracellular microelectrode recordings and signal analysis}

Extracellular microelectrode recordings and signal analysis were performed as described [14]. Network activity was recorded on multi-electrode arrays (MEAs) (Multi Channel Systems) with 64 titanium nitride electrodes (30 $\mu \mathrm{m}$ diameter and $200 \mu \mathrm{m}$ spacing) at $37{ }^{\circ} \mathrm{C}$ using sterile conditions. Signals from all 64 electrodes were simultaneously sampled at $25 \mathrm{kHz}$, visualized and stored using the standard software MC-Rack (Multi Channel Systems). Spike and burst detection was performed offline using specialized software (SPANNER 2.0, Result, Germany).

\section{Statistical analysis}

Statistical analysis was performed using spreadsheet (Microsoft Excel) and Prism (Graphpad) software. Multiple group analyses were conducted using two-way analysis of variance (ANOVA) and Bonferroni or Dunnett's post hoc test, and comparison of two groups using the two-tailed $t$-test. $P$-values $<0.05$ were considered significant.

\section{Results \\ DMF protects cells from oxidative stress by enhancing Nrf2 abundance and nuclear translocation in a time- and concentration-dependent manner}

Pre-incubation with $10 \mu \mathrm{M}$ DMF, but not MMF, for $24 \mathrm{~h}$ protected primary cortical cultures and hippocampal HT22 cells from oxidative glutamate toxicity (Figure 1A) in line with the increase in cellular glutathione levels seen in both the absence and presence of glutamate (Figure 1B). The protective effect was concentrationdependent for DMF (Figure 1C), but not for MMF at $24 \mathrm{~h}$ (Figure 1D). MMF protection took longer to develop and only became evident after incubation for $96 \mathrm{~h}$ (Figure 1E), whereas the effect of DMF was already maximal at $24 \mathrm{~h}$ (Figure 1F). When added together with glutamate, DMF exacerbated toxicity (Figure 1F) in line with the observation that DMF alone reduced cellular glutathione already after a $1 \mathrm{~h}$ exposure (Figure 1G). The transcription factor Nrf2 plays a key role in regulating the expression of proteins involved in GSH metabolism. DMF increased Nrf2 protein abundance at concentrations below $10 \mu \mathrm{M}$ as shown by immunoblotting of nuclear fractions (Figure $1 \mathrm{H}$ ) and nuclear localization as shown by immunocytochemistry after $24 \mathrm{~h}$ of $10 \mu \mathrm{M}$ DMF treatment (Figure 1I). The previously reported [17] inhibition of NF- $\mathrm{KB}$ translocation to the nucleus by DMF was not evident in these cells. In addition, TNFa, which served as a positive control, induced nuclear translocation of NF- $\mathrm{kB}$, which was not blocked by DMF (Figure 1J). This suggested that the protective effect of DMF in these cells is mainly mediated via the Nrf2-glutathione pathway. In line with this, we observed no increase in glutathione content in response to DMF in fibroblasts lacking Nrf2 (Figure 1K).

\section{DMF protection involves glutathione recycling}

DMF increased the mRNA abundance of various genes involved in the antioxidant response in HT22 cells including the enzymes glutamate-cysteine ligase (GCLC), NQO1, and peroxiredoxin 1, as well as the system Xcsubunit $\mathrm{xCT}$ while glutathione $\mathrm{S}$-transferase 1 and heme-oxygenase 1 were downregulated. In primary cortical cultures, only $\mathrm{xCT}$ and $\mathrm{NQO} 1$ were upregulated by DMF (Figure 2A). We then asked whether inhibition of the function of the most upregulated transcripts, $\mathrm{xCT}$ 


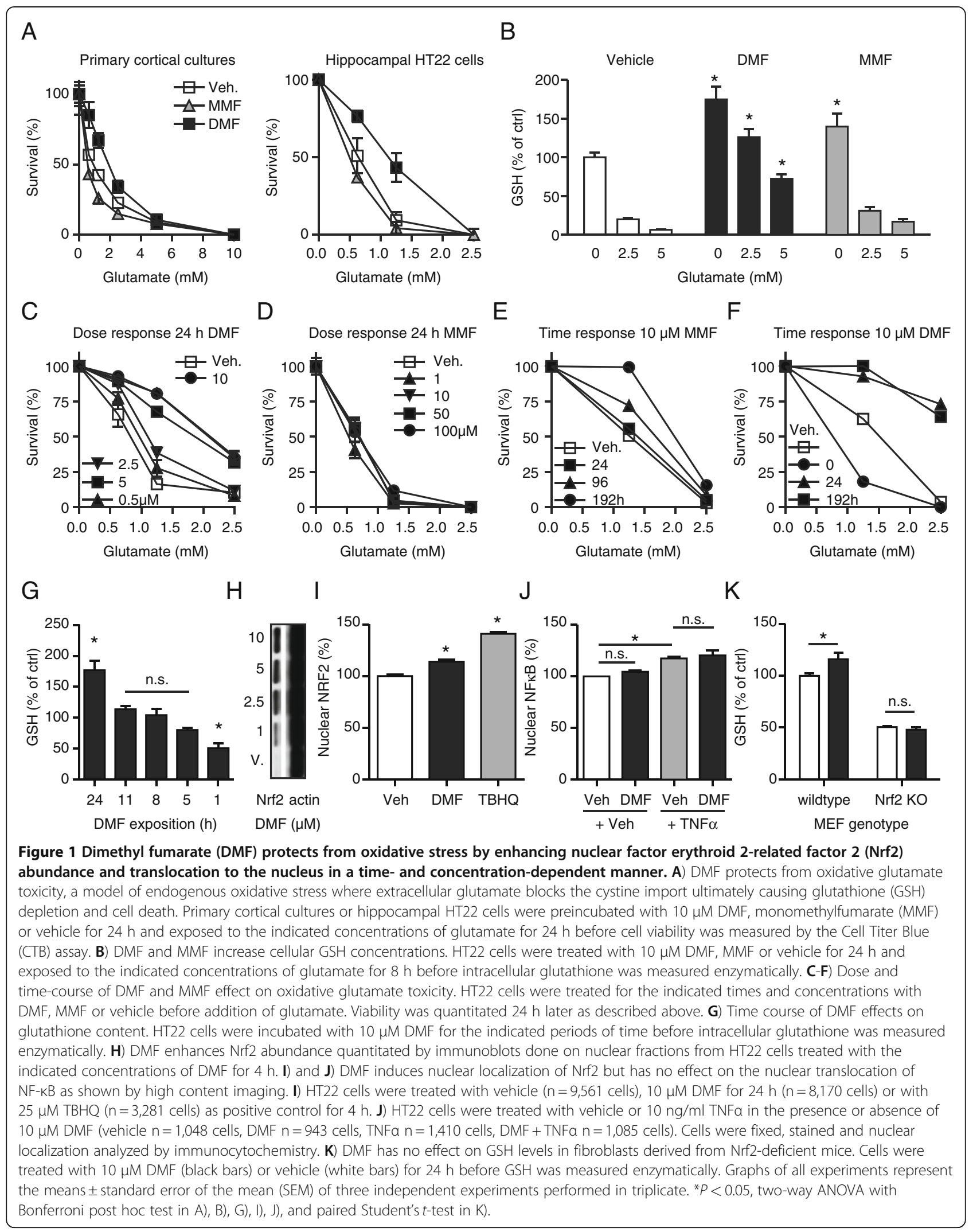




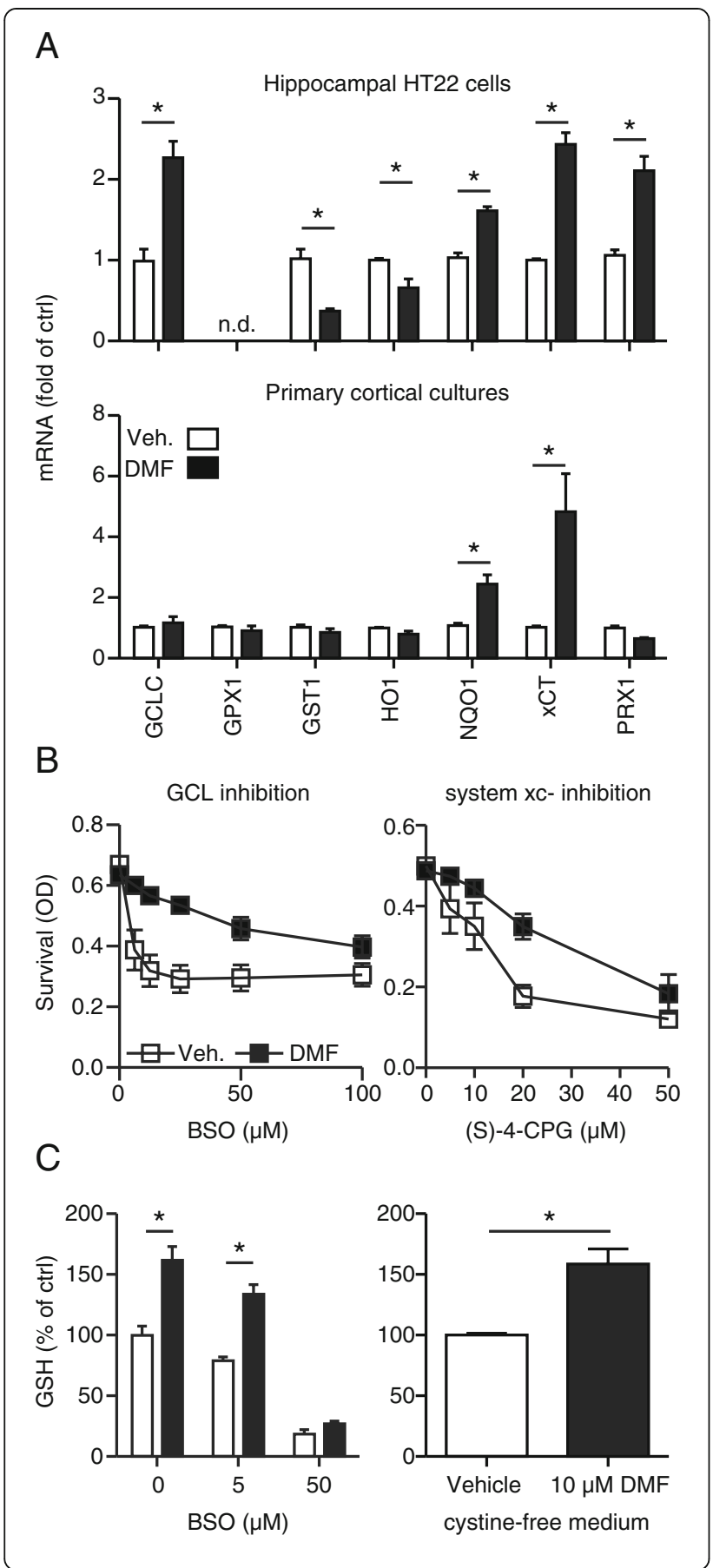

and GCLC with S4-CPG and buthionine sulfoximine (BSO), respectively, abolished the protective activity of DMF. However, DMF was capable of protecting against both compounds (Figure 2B). DMF was also still able to raise glutathione levels when GCLC was inhibited or when system Xc- activity was abrogated by incubation in cysteine-free medium (Figure 2C). Therefore, DMF can still exert protection in neuronal cells when de novo
Figure 2 Dimethyl fumarate (DMF)-mediated protection in neuronal cells involves glutathione recycling. A) DMF treatment induces mRNA expression of transcripts involved in the antioxidant response. Cells were treated for $24 \mathrm{~h}$ with $10 \mu \mathrm{M}$ DMF or vehicle and mRNA quantitated by real-time PCR using $\beta$-actin and hprt as endogenous controls. B) DMF protects from inhibition of GCLC by BSO and inhibition of system Xc-by (S)-4- carboxyphenylglycine (s-4-CPG). HT22 cells were treated for $24 \mathrm{~h}$ with $10 \mu \mathrm{M}$ DMF or vehicle and exposed to the indicated concentrations of s-4-CPG or $\mathrm{BSO}$ for another $24 \mathrm{~h}$ before cell viability was measured by the CTB assay. C) DMF still elevates cellular GSH when GSH synthesis is blocked by GCLC inhibition by BSO or system Xc - inhibition by incubation in cystine-free medium. HT22 cells were treated for $24 \mathrm{~h}$ with $10 \mu \mathrm{M}$ DMF (black bars) or vehicle (white bars) and then exposed to the indicated concentrations of BSO or to cystine-free medium for another 24 or $4 \mathrm{~h}$ respectively before intracellular GSH was measured enzymatically. Graphs of all experiments represent the means \pm standard error of the mean (SEM) of three independent experiments performed in triplicate. ${ }^{*} P<0.05$, paired Student's $t$-test.

glutathione synthesis is blocked, suggesting that it enhances glutathione recycling.

Neuroprotective concentrations of DMF suppress cytokine production in activated splenocytes from two different mouse strains without exerting effects on viability

We then assessed the effects of $10 \mu \mathrm{M}$ DMF, which was clearly neuroprotective, on the viability and immune response of primary mouse splenocytes isolated from C57BL6 and SJL mice. Apoptosis was quantitated by flow cytometry using Annexin $\mathrm{V}$ translocation and cell death by 7-AAD staining. While $100 \mu \mathrm{M}$ DMF increased dead and apoptotic cells in both strains, the effects of $10 \mu \mathrm{M}$ were indistinguishable from DMSO, which was used as the vehicle control (Figure 3A for C57BL6 mice and 3A' for SJL mice). Having established that neuroprotective concentrations of DMF were not harmful to primary splenocytes, we then quantitated TNF $\alpha$ production in response to stimulation with $1 \mu \mathrm{g} / \mathrm{ml}$ anti-CD3 for $48 \mathrm{~h}$. Here, $10 \mu \mathrm{M}$ DMF attenuated TNF $\alpha$ production in C57BL6-derived splenocytes (Figure 3B) and completely abolished it in SJL-derived splenocytes (Figure 3B'). We also analyzed direct changes in cytokine production in response to anti-CD3 stimulation $(0.5 \mu \mathrm{g} / \mathrm{ml}$ for $24 \mathrm{~h})$ with $10 \mu \mathrm{M}$ DMF or vehicle using ELISPOT technology. This showed a significantly reduced production of IL-2 and IL-17, but no changes in IL-4 and IL-5 in C57BL6 splenocytes (Figure 3C) and a reduction in IL-2, IL-4, IL-5, IL-6, and IL-17 production in SJL splenocytes (Figure 3C'). These data suggest that neuroprotective concentrations of DMF have a prominent immunomodulatory activity, which is more pronounced in the more immuneresponsive SJL background without increasing splenocyte apoptosis. 


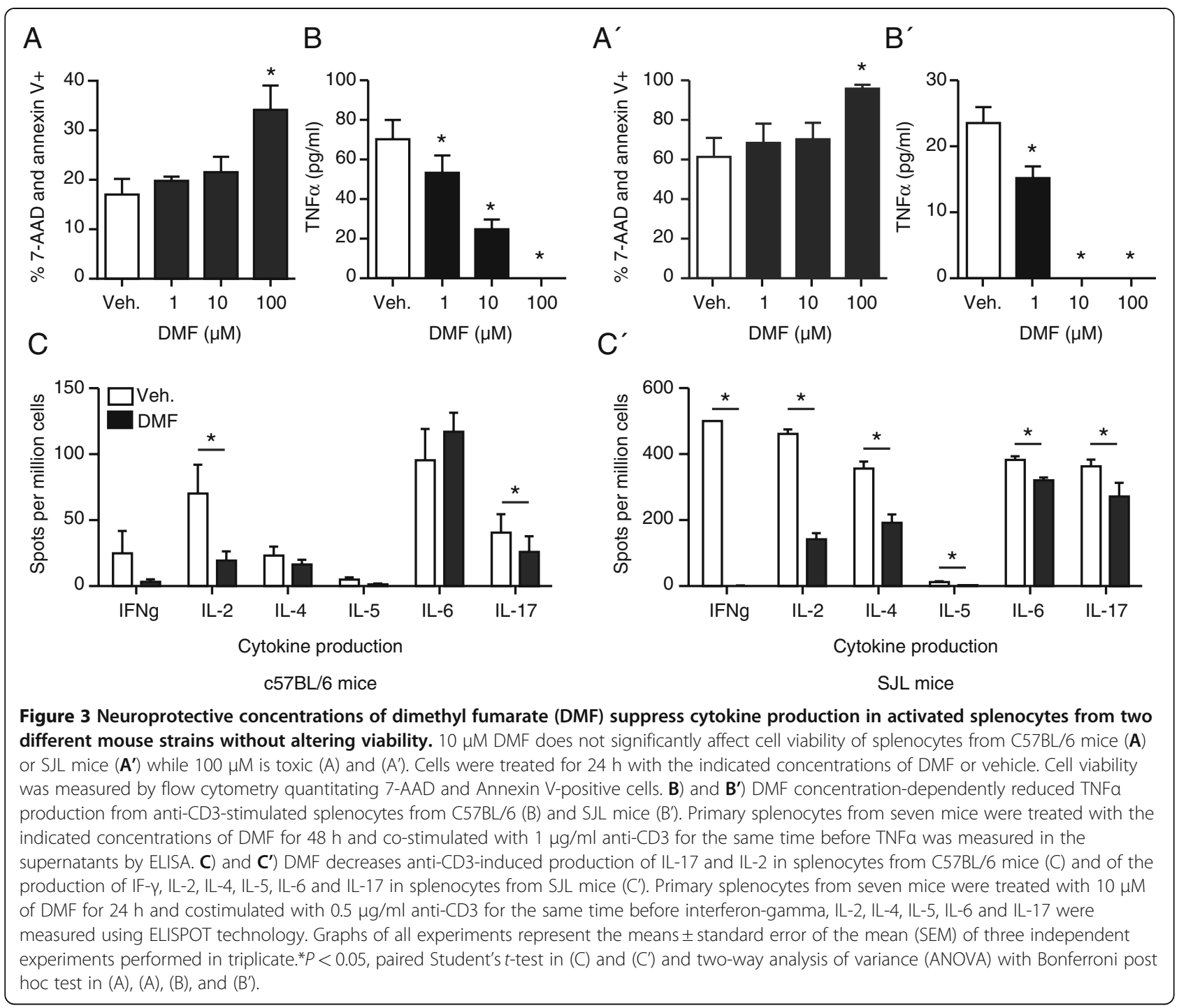

\section{DMF-treated neuronal cells but not splenocytes secrete neuroprotective GSH}

Pretreatment with $10 \mu \mathrm{M}$ DMF for $24 \mathrm{~h}$ increased the GSH content in both HT22 cells and splenocytes to a similar degree. However, DMF maintained the increase in GSH in cystine-free medium only in HT22 cells but not in splenocytes suggesting differences in GSH secretion or recycling (Figure 4A). We further sought to determine whether these DMF-mediated changes in GSH content and viability of neuronal cells alter the viability of splenocytes and vice versa. HT22 cells and splenocytes were treated with $10 \mu \mathrm{M}$ DMF or vehicle for $24 \mathrm{~h}$. DMF was then removed and the cells were incubated in DMF-free medium to allow accumulation of secreted GSH (or other DMF-induced secreted substances) for another $24 \mathrm{~h}$ followed by transfer of the conditioned medium to untreated cultures for an additional $24 \mathrm{~h}$.
The medium conditioned by DMF-pretreated HT22 cells elevated total cellular GSH in new HT22 cells while the medium preconditioned by DMF-pretreated splenocytes did not (Figure 4B). Furthermore, medium preconditioned by DMF-pretreated HT22 cells also protected new HT22 cells from oxidative glutamate toxicity (Figure 4C) but had no effect on the viability of unstimulated splenocytes (Figure 4D). It was not possible to do the reverse experiment using DMF-preconditioned splenocyte medium on HT22 cells, as the oxidativeglutamate-toxicity assay does not work in splenocyte medium.

These data suggest that while both neuronal and immune cells raise intracellular GSH levels upon DMF treatment, only neuronal cells secrete this GSH into the extracellular space where it can protect other neuronal cells. Apparently splenocytes do not secrete GSH and do 


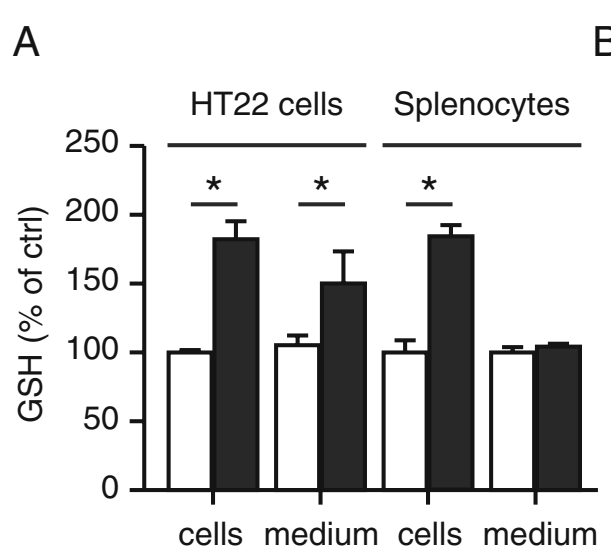

B

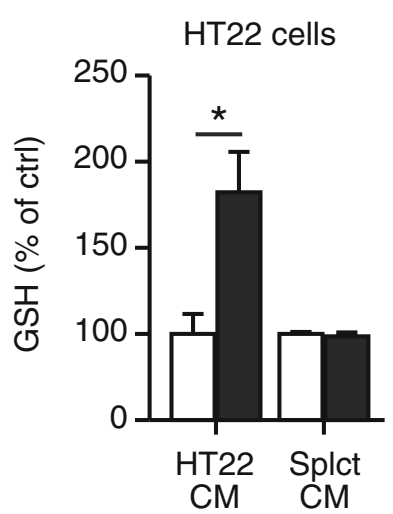

C

D
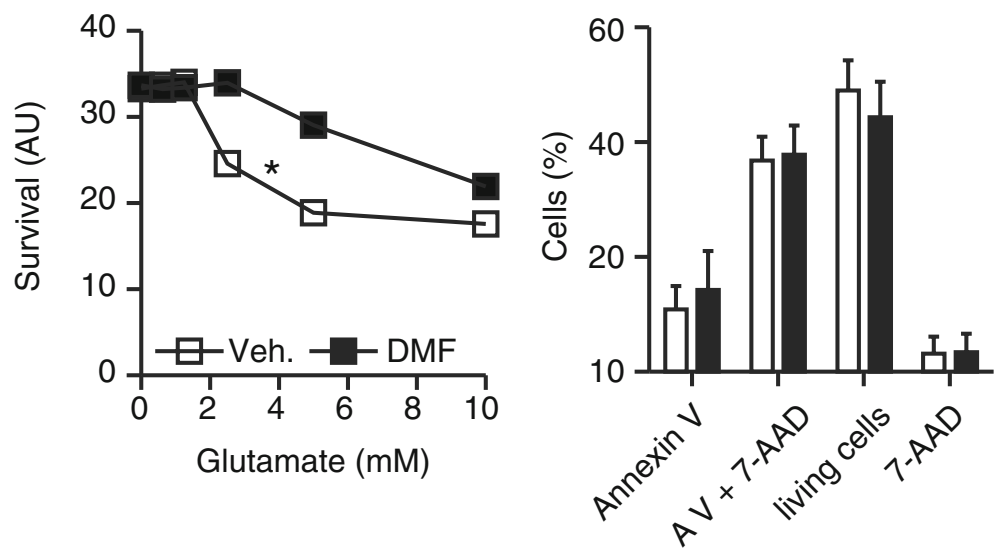

Figure 4 Dimethyl fumarate (DMF)-treated neuronal cells but not splenocytes secrete neuroprotective glutathione (GSH). A) HT22 cells and splenocytes show increased intracellular glutathione after $24 \mathrm{~h}$ pretreatment with $10 \mu \mathrm{M}$ DMF, but only HT22 cells release glutathione into the extracellular space. GSH was quantitated enzymatically and normalized to cellular protein content and vehicle-treated cells, respectively. Released GSH in the medium was quantitated after $4 \mathrm{~h}$ incubation in cystine-free medium following a $24 \mathrm{~h}$ incubation in medium supplemented with $10 \mu \mathrm{M}$ DMF (black bars) or vehicle (white bars). B) HT22 cells treated with conditioned medium (CM) from HT22 cells but not splenocytes take up released GSH and $\mathbf{C}$ ) are protected from glutamate toxicity. HT22 cells were treated for $24 \mathrm{~h}$ with conditioned medium before addition of the indicated concentrations of glutamate for another $24 \mathrm{~h}$. Viability was quantitated by the CTB assay. (D) Viability of unstimulated splenocytes quantitated by flow cytometry using Annexin $V$ and 7AAD staining is unaffected by HT22 conditioned medium. Graphs of all experiments represent the means \pm standard error of the mean (SEM) of three independent experiments performed in triplicate. ${ }^{*} P<0.05$, paired Student's ttest for all assays except two-way analysis of variance (ANOVA) with Bonferroni post hoc test for (C).

not benefit from GSH released by neuronal cells, at least under the conditions employed here.

\section{No effect of DMF on the network activity of primary dissociated cortical cultures grown on multi-electrode arrays}

MEAs allow a highly sensitive and reproducible assessment of network activity by culturing dissociated cortical cells directly on-chip (Figure 5A). In these cultures, spontaneous electrical activity and signal propagation of evolving neuronal networks can be observed non-invasively in real time at 64 electrodes embedded into the chip. After three weeks in vitro, networks exhibit regular bursting activity consisting of single spikes or bursts, which can be measured quantitatively by the spike and burst rate per minute and the inter-burst interval (Figure 5B) [14,18]. Mature neuronal cultures derived from dissociated cortical cells exhibited highly synchronous network activity on MEAs (Figure 5C left panel), but incubation with 10 or $100 \mu \mathrm{M}$ DMF for $30 \mathrm{sec}, 1 \mathrm{~h}$ or $24 \mathrm{~h}$ did not change the spike or burst frequency, inter-burst interval or synchronicity of these cultures (Figure 5D). We conclude that even excessive concentrations of DMF have no effect on neuronal network activity measured with MEAs.

\section{Discussion}

Our main finding is that DMF at low concentrations protects neuronal cells from oxidative stress by elevating 


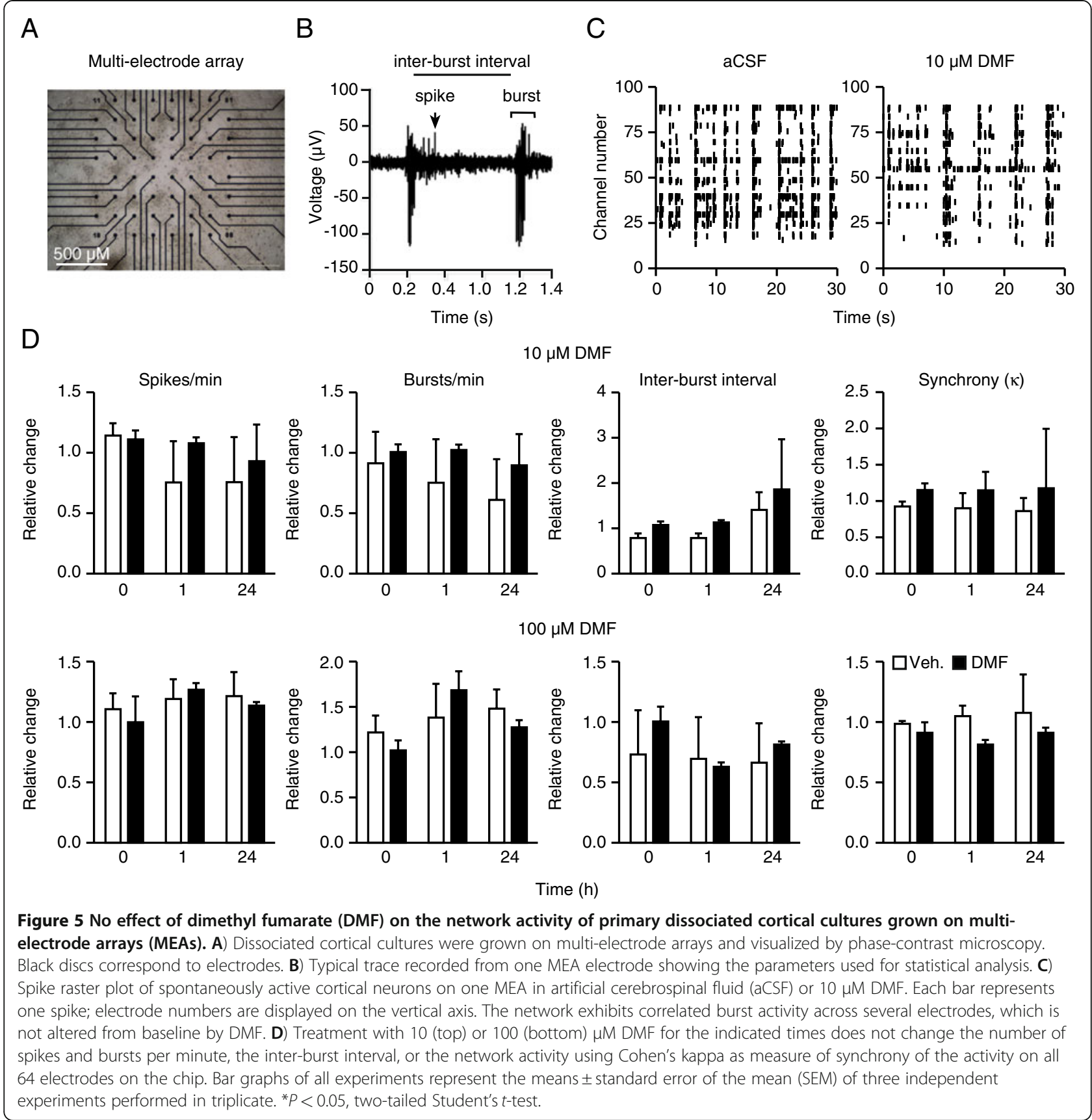

cellular glutathione, and that similar concentrations also reduce production of proinflammatory cytokines from splenocytes. In our experiments, DMF protection needed less time to develop than protection induced by MMF. The induction of the antioxidant response leading to glutathione synthesis seems to be the consequence of an initial and short-lived oxidative stress, since DMF decreased the glutathione content immediately after its addition to the cells. Most likely DMF as an unsaturated carboxylic acid ester initially binds and sequesters glutathione [19]. The long-term effect of DMF in neuronal cells is most probably mediated via Nrf2 as other reported mechanisms such as the inhibition of the nuclear translocation of NF- $\mathrm{KB}$ [19] were not evident in these cells and because the increase in GSH synthesis was abolished in cells lacking Nrf2.

On the mRNA level, the most prominently upregulated transcript in HT22 cells and primary cortical cultures was $\mathrm{xCT}$, the functional subunit of system Xc-, which is tightly involved in glutathione homeostasis (reviewed in [13]). DMF, however, also raised the glutathione content when system Xc- activity was inhibited 
pharmacologically or by incubation in cysteine-free medium, which suggests enhanced glutathione recycling through a mechanism that is as yet unknown.

We observed no upregulation of IL- 4 and IL-5 but a significant downregulation of TNF $\alpha$, IL-2 and IL-17 in DMF-treated anti-CD3-stimulated primary mouse splenocytes from C57BL/6 mice, and additionally IL-4, IL-5, IL-6, IL-17 and IFN $\gamma$ downregulation in DMF-treated splenocytes from SJL mice, which are a more immuneresponsive strain. It has to be kept in mind that in these experiments we analyzed the direct effect of short-term, low-concentration DMF treatment on unsorted splenocytes without priming by antigen-presenting cells. A previous study reported that treatment with $70 \mu \mathrm{M}$ DMF augmented IL-4 production by $\mathrm{CD} 4+\mathrm{T}$ lymphocytes in vitro only when primed by dendritic cells but not anti-CD3/28 antibodies alone indicating the requirement of antigen-presenting cells for inducing a $\mathrm{TH} 2$ response $[8,19]$. Our data suggest an additional direct effect of DMF on immune cells which is different from its effect during the priming of a $\mathrm{T}$ cell response.

Our experiments using media that were preconditioned by DMF-pretreated neuronal cells indicate that both immune and neuronal cells display increased intracellular GSH after DMF treatment but only neuronal cells release this glutathione into the extracellular space where it raises the glutathione content of surrounding neuronal cells and protects them from oxidative stress. The same medium did not prevent unstimulated immune cells from dying which suggests that the death of these cells is not primarily mediated by oxidative stress or that they cannot take up the glutathione. Interestingly, despite elevation of cellular glutathione, DMF-pretreated splenocytes did not release glutathione and their medium did not increase the glutathione content of neuronal cells. As cystine influences the enzymatic glutathione assay employed here by disulfide exchange reactions with glutathione, the measurement of glutathione discharged into the medium was performed in cystine-free medium. Either splenocytes, in contrast to HT22 cells, do not secrete GSH or they have a heightened demand and use up the increased glutathione during the $4 \mathrm{~h}$ incubation in cystine-free medium. Alternatively, they might lack the machinery necessary to recycle glutathione.

Treatment with 10 or $100 \mu \mathrm{M}$ DMF did not alter the activity of primary cortical neurons plated on a multielectrode array indicating that it has no direct effects on neuronal function in vitro.

\section{Conclusions}

In summary, our findings demonstrate that DMF at low concentrations exerts protective effects on neuronal cells and diminishes the production of TNF- $\alpha$, IL-2, and IL17 in splenocytes from $\mathrm{C} 57 \mathrm{BL} / 6$ mice and the production of all cytokines measured in splenocytes from SJL mice. Although higher concentrations of DMF can cause cell death of primary splenocytes, this is probably not necessary for its immunomodulatory effect. These observations might be relevant for understanding the drug's presumed mechanism of action as we assume that the active metabolite MMF has similar effects that merely need a longer time to develop.

\section{Abbreviations}

7-AAD: 7-amino-actinomycin D; ANOVA: analysis of variance; BSO: buthionine sulfoximine; CTB: Cell Titer Blue; DMF: dimethyl fumarate; DMSO: dimethyl sulfoxide; ELISA: enzyme-linked immunosorbent assay; GCLC: glutamatecysteine ligase; GSH: glutathione; IL: Interleukin; IFN- $\gamma$ : cytokine interferon gamma; MEA: multi-electrode array; MMF: monomethylfumarate; MS: multiple sclerosis; NF-kB: nuclear factor kappa B; Nrf2: erythroid 2-related factor 2; NQO1: NADPH-quinone-oxidoreductase-1; PCR: polymerase chain reaction; S4-CPG: (S)-4- carboxyphenylglycine; SEM: standard error of the mean; TBHQ: tert-butyl hydroquinone; TNFa: tumor necrosis factor alpha.

\section{Competing interests}

This work was supported by an unrestricted research grant by Biogen Idec to $\mathrm{AM}$, and grants from the Deutsche Forschungsgemeinschaft to $\mathrm{HHH}(\mathrm{Ho}$ 4392/1-1) and the Heinrich Heine University to PA (701220973). HHH was also supported by the Deutsche Multiple Sklerose Gesellschaft and the

Strategischer Forschungsfonds der Heinrich Heine Universität. HPH received honoraria with approval by the Rector of Heinrich-Heine University from Bayer Healthcare $\mathrm{GmbH}$, Biogen Idec GmbH, Novartis Pharma GmbH, Teva Sanofi Aventis and Merck Serono GmbH for consulting and speaking at scientific symposia. Other than that the authors declare no competing interests.

\section{Author details}

${ }^{1}$ Department of Neurology, Heinrich-Heine-University Düsseldorf, Medical Faculty, Düsseldorf 40225, Germany. ${ }^{2}$ Department of Neurology, University Hospital of Ulm, Ulm 89081, Germany. ${ }^{3}$ Cellular Neurobiology Laboratory, Salk Institute for Biological Studies, La Jolla, CA 92037, USA. ${ }^{4}$ Neurologische Klinik der Heinrich-Heine-Universität Düsseldorf, Moorenstr. 5, D-40225, Düsseldorf 40225, Germany.

\section{Authors' contributions}

PA and AM conceived the study and participated in its design and coordination, performed the statistical analysis and wrote the manuscript. IB and Al carried out cell viability assays, glutathione measurements, reporter assays, and quantitative real-time PCR experiments. $\mathrm{CZ}$ and HHH performed and analyzed the ELISPOT assays. AKM performed the TNFa ELISAs. ZK helped with the reporter assays and cell viability assays. $\mathrm{NH}$ and $\mathrm{DL}$ performed the translocation assays. JL participated in the study design. PM performed the immunoblotting. NG and KQ performed the MEA analysis. All authors critically revised and approved the final manuscript.

Received: 11 February 2012 Accepted: 18 June 2012 Published: 7 July 2012

\section{References}

1. Compston A, Coles A: Multiple sclerosis. Lancet 2008, 372:1502-1517. doi:10.1016/S0140-6736(08)61620-7.

2. Gonsette RE: Neurodegeneration in multiple sclerosis: the role of oxidative stress and excitotoxicity. J Neurol Sci 2008, 274:48-53. doi:10.1016/j.jns.2008.06.029.

3. Nave K-A, Trapp BD: Axon-glial signaling and the glial support of axon function. Annu Rev Neurosci 2008, 31:535-561. doi:10.1146/annurev. neuro.30.051606.094309.

4. Kappos L, Gold R, Miller DH, MacManus DG, Havrdova E, Limmroth V, Polman CH, Schmierer K, Yoursry TA, Yang M, Eraksoy M, Meluzinova $E_{\text {, }}$ Rektor I, Dawson KT, Sandrock AW, O’Neill GN, bg-12 Phase Ilb Study Investigators: Efficacy and safety of oral fumarate in patients with relapsing-remitting multiple sclerosis: a multicentre, randomised, double-blind, placebo-controlled phase Ilb study. Lancet 2008, 372:1463-1472. doi:10.1016/S0140-6736(08)61619-0. 
5. Gold R, Kappos L, Bar-Or D, Arnold D, Giovannoni G, Selmaj K, Yang M, Dawson K: In Clinical efficacy of BG-12, an oral therapy, in relapsing-remitting multiple sclerosis: data from the phase 3 DEFINE trial, In Proceedings of the $5^{\text {th }}$ joint triennial congress of the European and America's Committees for treatment and research in multiple sclerosis (ECTRIMS/ACTRIMS). Edited by Thompson A. Multiple Sclerosis Journal 2011. Amsterdam: October 19-22 2011. 17:S9-S52.

6. Vandermeeren $M$, Janssens $S$, Wouters $H$, Borghmans I, Borgers $M$, Beyaert $R$, Geysen J: Dimethylfumarate is an inhibitor of cytokine-induced nuclear translocation of NF-kappa B1, but not RelA in normal human dermal fibroblast cells. J Invest Dermatol 2001, 116:124-130. doi:10.1046/j.1523-1747.2001.00211.x.

7. Treumer F, Zhu K, Gläser R, Mrowietz U: Dimethylfumarate is a potent inducer of apoptosis in human T cells. J Invest Dermatol 2003, 121:1383-1388. doi:10.1111/j.1523-1747.2003.12605.x.

8. de Jong R, Bezemer AC, Zomerdijk TP, van de Pouw-Kraan T, Ottenhoff TH, Nibbering PH: Selective stimulation of T helper 2 cytokine responses by the anti-psoriasis agent monomethylfumarate. Eur J Immunol 1996, 26:2067-2074. doi:10.1002/eji.1830260916.

9. Linker RA, Lee DH, Ryan S, van Dam AM, Conrad R, Bista P, Zeng W, Hronowsky X, Buko A, Chollate S, Ellrichmann G, Brück W, Dawson K, Goelz S, Wiese S, Scannevin RH, Lukashev M, Gold R: Fumaric acid esters exert neuroprotective effects in neuroinflammation via activation of the Nrf2 antioxidant pathway. Brain 2011, 134:678-692. doi:10.1093/brain/awq386.

10. Lin SX, Lisi L, Russo Dello C, Polak PE, Sharp A, Weinberg G, Kalinin S, Feinstein DL: The anti-inflammatory effects of dimethyl fumarate in astrocytes involve glutathione and haem oxygenase-1. ASN Neuro 2011, 3:75-84. doi:10.1042/AN20100033.

11. Thiessen A, Schmidt MM, Dringen R: Fumaric acid dialkyl esters deprive cultured rat oligodendroglial cells of glutathione and upregulate the expression of heme oxygenase 1. Neurosci Lett 2010, 475:56-60. doi:10.1016/..neulet.2010.03.048.

12. Schmidt MM, Dringen R: Fumaric acid diesters deprive cultured primary astrocytes rapidly of glutathione. Neurochem Int 2010, 57:460-467. doi:10.1016/j.neuint.2010.01.006.

13. Albrecht P, Lewerenz J, Dittmer S, Noack R, Maher P, Methner A: Mechanisms of oxidative glutamate toxicity: the glutamate/cystine antiporter system xc- as a neuroprotective drug target. CNS Neurol Disord Drug Targets 2010, 9:373-382

14. Steinbeck JA, Henke N, Opatz J, Gruszczynska-Biegala J, Schneider L, Theiss S, Hamacher N, Steinfarz B, Golz S, Brüstle O, Kuznicki J, Methner A: Storeoperated calcium entry modulates neuronal network activity in a model of chronic epilepsy. Exp Neurol 2011, 232:185-194. doi:10.1016/j.expneurol.2011.08.022

15. Lewerenz J, Albrecht P, Tien M-LT, Henke N, Karumbayaram S, Kornblum HI, Wiedau-Pazos M, Schubert D, Maher P, Methner A: Induction of Nrf2 and $\mathrm{xCT}$ are involved in the action of the neuroprotective antibiotic ceftriaxone in vitro. I Neurochem 2009, 111:332-343. doi:10.1111/j.1471-4159.2009.06347.x.

16. Hofstetter HH, Lühder F, Toyka KV, Gold R: IL-17 production by thymocytes upon CD3 stimulation and costimulation with microbial factors. Cytokine 2006, 34:184-197. doi:10.1016/j.cyto.2006.04.014.

17. Loewe R, Holnthoner W, Gröger M, Pillinger M, Gruber F, Mechtcheriakova $D$, Hofer E, Wolff $K$, Petzelbauer P: Dimethylfumarate inhibits TNF-induced nuclear entry of NF-kappa B/p65 in human endothelial cells. J Immunol 2002, 168:4781-4787.

18. Gramowski A, Jügelt K, Stüwe S, Schulze R, McGregor GP, WartenbergDemand A, Loock J, Schröder O, Weiss DG: Functional screening of traditional antidepressants with primary cortical neuronal networks grown on multielectrode neurochips. Eur J Neurosci 2006, 24:455-465. doi:10.1111/j.1460-9568.2006.04892.x.

19. Ghoreschi K, Bruck J, Kellerer C, Deng C, Peng H, Rothfuss O, Hussain RZ, Gocke AR, Respa A, Glocova I, Valtcheva N, Alexander E, Feil S, Feil R, SchulzeOsthoff K, Rupec RA, Lovett-Racke AE, Dringen R, Racke MK, Röcken M: Fumarates improve psoriasis and multiple sclerosis by inducing type II dendritic cells. J Exp Med 2011, 208:2291-2303. doi:10.1084/jem.20100977.

doi:10.1186/1742-2094-9-163

Cite this article as: Albrecht et al:: Effects of dimethyl fumarate on neuroprotection and immunomodulation. Journal of Neuroinflammation 2012 9:163.

\section{Submit your next manuscript to BioMed Central and take full advantage of:}

- Convenient online submission

- Thorough peer review

- No space constraints or color figure charges

- Immediate publication on acceptance

- Inclusion in PubMed, CAS, Scopus and Google Scholar

- Research which is freely available for redistribution 